\title{
Hofstadter butterflies of carbon nanotubes: Pseudofractality of the magnetoelectronic spectrum
}

\author{
Norbert Nemec and Gianaurelio Cuniberti \\ Institute for Theoretical Physics, University of Regensburg, D-93040 Regensburg, Germany \\ (Received 28 June 2006; revised manuscript received 29 August 2006; published 23 October 2006)
}

\begin{abstract}
The electronic spectrum of a two-dimensional square lattice in a perpendicular magnetic field has become known as the Hofstadter butterfly [Hofstadter, Phys. Rev. B 14, 2239 (1976).]. We have calculated quasi-onedimensional analogs of the Hofstadter butterfly for carbon nanotubes (CNTs). For the case of single-wall CNTs, it is straightforward to implement magnetic fields parallel to the tube axis by means of zone folding in the graphene reciprocal lattice. We have also studied perpendicular magnetic fields which, in contrast to the parallel case, lead to a much richer, pseudofractal spectrum. Moreover, we have investigated magnetic fields piercing double-wall CNTs and found strong signatures of interwall interaction in the resulting Hofstadter butterfly spectrum, which can be understood with the help of a minimal model. Ubiquitous to all perpendicular magnetic field spectra is the presence of cusp catastrophes at specific values of energy and magnetic field. Resolving the density of states along the tube circumference allows recognition of the snake states already predicted for nonuniform magnetic fields in the two-dimensional electron gas. An analytic model of the magnetic spectrum of electrons on a cylindrical surface is used to explain some of the results.
\end{abstract}

DOI: 10.1103/PhysRevB.74.165411 PACS number(s): 73.63.Fg, 73.22.-f, 73.43.-f, 73.43.Qt

\section{INTRODUCTION}

The availability of new materials for nanoelectronic research allows for a detailed test of the emergence of the quantum physical nature of electrons, via transport or optical measurements. Carbon nanotubes ${ }^{2-7}$ (CNTs) are an example of a very peculiar electronic material, due to the extreme confinement of electrons on their $\pi$-conjugated "walls". In these systems, many mesoscopic phenomena such as singleelectron charging, ${ }^{8}$ and conductance quantization, ${ }^{9}$ as well as effects typical for semiconductor physics like $s$-like excitons, ${ }^{10}$ can be observed already at room temperature.

Since the prediction of band structure effects of carbon nanotubes in parallel external fields by Ajiki and Ando in $1993,{ }^{11}$ it took only a few years until clear hallmarks of a single quantum flux being tethered within a tube section were found experimentally in optical ${ }^{12}$ and transport $^{13,14}$ measurements. For magnetic fields perpendicular to the CNT axis, theoretical predictions were made shortly after, first using a perturbative approach around the Fermi energy, ${ }^{15}$ and later also using a tight-binding model. ${ }^{16,17}$ Only recently, a first experimentally accessible effect of perpendicular magnetic fields-anomalous magnetoconductance-was predicted $^{18}$ and observed. ${ }^{19}$ A very similar effect for strong electric fields has also been found by numerical studies ${ }^{20}$ and has yet to be confirmed experimentally. The use of magnetic fields to further investigate the interplay between elastic mean free path, phase coherent length, and electron-electron interaction was also successfully adopted. ${ }^{21-23}$

From the purely theoretical perspective, carbon nanotubes in strong perpendicular magnetic fields represent a very interesting case of study. Closely related to graphene, their energy spectrum shows strong similarities with that of the two-dimensional (2D) honeycomb lattice, ${ }^{24-26}$ which again forms a variation of the fractal butterflylike pattern discovered by Hofstadter ${ }^{1}$ in 1976 and studied intensely since that time from various points of view. ${ }^{27-32}$ Yet the quasi-1D nature and the curvature of CNTs set their energy spectra clearly apart from the fractal and perfectly periodic images obtained in 2D lattices.

In this paper, we will describe a method of computing and visualizing the spectrum of carbon nanotubes (for a prototypical example see Fig. 1). This method will be demonstrated on a number of single- and double-wall CNTs (SWCNTs and DWCNTs) of different chirality and diameter. The study of the local distribution of the spectral density will shed some light on the relation between the spectrum of a planar sheet of graphene and that of a CNT, strongly affected by curvature and finite size. A closer look at the spectrum will reveal the presence of cusp catastrophes, which are closely related to the quenching of the Bloch state velocity, induced by a magnetic field.

For magnetic fields parallel to the tube axis, the natural unit is that of one flux quantum per tube cross section $r^{2} \pi$

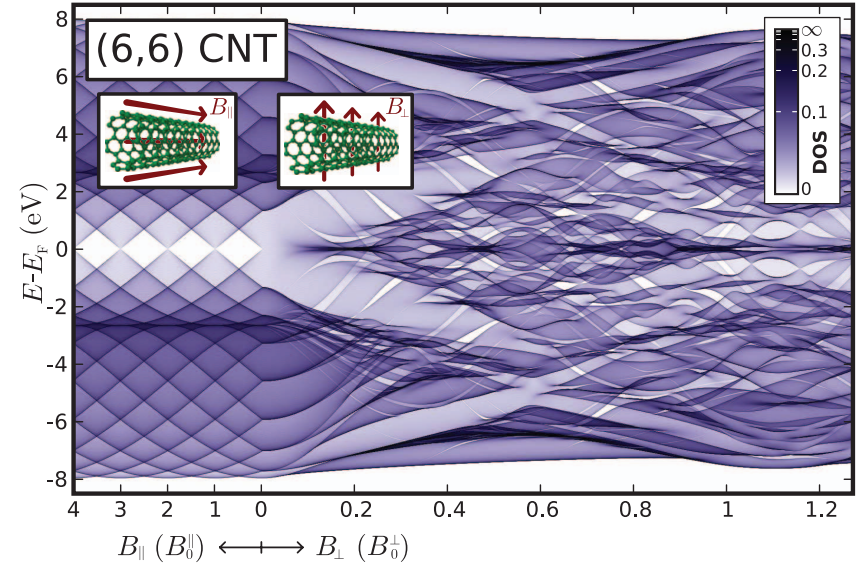

FIG. 1. (Color online) Density of states of a $(6,6)$ CNT in dependence on an external magnetic field parallel (left) or perpendicular (right) to the tube axis. For every value of the magnetic field, the DOS is unity normalized over energy. The units $B_{0}^{\|}=\Phi_{0} / r^{2} \pi$ and $B_{0}^{\perp}=\Phi_{0} / A_{\text {plaquette }}$ (see text) are scaled such that the physical field scale is the same for both segments of the plot. 


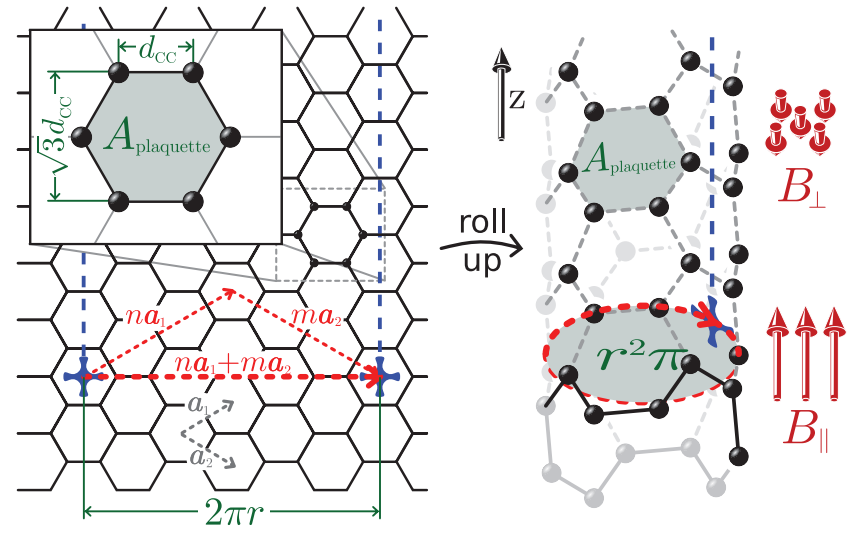

FIG. 2. (Color online) The structure of a CNT [here, a (3,3)$\mathrm{CNT}$ ]: the hexagonal lattice of a graphene sheet is rolled up in such a way that the chiral vector $(n, m)$ becomes the circumference of the resulting cylinder. Magnetic fields parallel to the tube axis pierce the tube cross section $r^{2} \pi$, while perpendicular magnetic fields pierce the wall made up from hexagonal plaquettes.

(see Fig. 2). For a general $(n, m) \mathrm{CNT}$ the tube radius can be obtained with simple geometrical arguments $2 \pi r$ $=\sqrt{3 m^{2}+3 n^{2}+3 m n} d_{\mathrm{CC}}$, where $d_{\mathrm{CC}}=1.42 \AA$ denotes the carbon-carbon distance. This immediately gives the parallel magnetic field $B_{0}^{\|}$needed to pierce one flux quantum $\Phi_{0}=h / e$ through an $(n, m)$ CNT. For perpendicular magnetic fields, the scale is ruled by the field necessary to enclose a flux through a single benzene ring, the plaquette of graphene and carbon nanotubes of area $A_{\text {plaquette }}$ $=3 \sqrt{3 / 4} d_{\mathrm{CC}}^{2} \sim 5.24 \AA^{2}$. Because of this extremely small area we obtain $B_{0}^{\perp}=\Phi_{0} / A_{\text {plaquette }}=79 \times 10^{3} \mathrm{~T}$, which is, of course, out of experimental reach. ${ }^{33}$ It is straightforward to get the relation between the parallel and perpendicular field scales as

$$
B_{0}^{\|}=\frac{\Phi_{0}}{r^{2} \pi}=\frac{2 \sqrt{3} \pi}{m^{2}+n^{2}+m n} B_{0}^{\perp} .
$$

For a typical SWCNT with $\sim 1 \mathrm{~nm}$ diameter, this gives a value of $B_{0}^{\|} \approx 5 \times 10^{3} \mathrm{~T}$. It is thus understandable that multiwall CNTs (MWCNTs) present a more interesting object for magnetic field experiments: For a typical MWCNT with a diameter of $20 \mathrm{~nm}$, as a matter of fact, one can already observe the first Aharonov-Bohm oscillations accessible at around $12 \mathrm{~T}$ parallel fields. ${ }^{13}$ As shown in this work, however, even for perpendicular fields low-field signatures could be visible within experimentally accessible field ranges if one takes into account the external shell of a MWCNT.

This paper is organized as follows. We first give definitions, introduce the method of computation and visualization, and point toward general features observable in quasi-1D systems. In Sec. III, we then do a systematic study of SWCNTs, including an analytic model and a detailed view of the range of experimentally accessible fields. In Sec. IV, we proceed with an analysis of the effects of the interwall interaction in DWCNTs on the magnetic spectrum and introduce a minimal model, closing with a discussion of the results in the last secion.

\section{DEFINITIONS, METHODS, AND OBSERVABLES}

Lattice electrons in arbitrary external magnetic fields. All numerical calculations in this work are based on a tightbinding Hamiltonian of the form

$$
\mathcal{H}(\boldsymbol{B})=\sum_{i} \varepsilon_{i} c_{i}^{\dagger} c_{i}-\sum_{\langle i, j\rangle} \gamma_{i j}(\boldsymbol{B}) c_{i}^{\dagger} c_{j}
$$

where the indices denote the atomic orbitals. For the singleorbital approximation used hereafter, these coincide with the label of the atom so that $\mathcal{H}$ can be represented by means of the matrix elements $\mathcal{H}_{\boldsymbol{r}_{i}, \boldsymbol{r}_{j}}$ between $\pi$ orbitals centered on the atom $i$ and $j$ at the position $\boldsymbol{r}_{i}\left(\boldsymbol{r}_{j}\right)$ of the CNT molecular network.

An external magnetic field is implemented using the Peierls substitution: ${ }^{34}$ Based on the principle of minimal coupling $\boldsymbol{p} \rightarrow \boldsymbol{p}-e \boldsymbol{A}$, the effect of a magnetic field $\boldsymbol{B}=\operatorname{rot} \boldsymbol{A}$ is absorbed in the translation operator $\mathcal{T}(\boldsymbol{R})=\exp \frac{\mathrm{i}}{\hbar}(\boldsymbol{p}-e \boldsymbol{A}) \cdot \boldsymbol{R}$. In the tight-binding Hamiltonian, this is reflected by representing the hopping matrix elements $\gamma_{i j}=\left\langle\Psi_{i}|\mathcal{H}| \Psi_{j}\right\rangle$ between two $\pi$ orbitals $\Psi_{i}$ and $\Psi_{j}$ localized at sites $\boldsymbol{r}_{i}$ and $\boldsymbol{r}_{j}$ as

$$
\gamma_{i j}(\boldsymbol{B})=\gamma_{i j}^{0} \exp \left(\mathrm{i} \frac{2 \pi}{\Phi_{0}} \int_{\boldsymbol{r}_{i}}^{\boldsymbol{r}_{j}} d \boldsymbol{r} \cdot \boldsymbol{A}_{\boldsymbol{B}}(\boldsymbol{r})\right) .
$$

The bare hopping at zero magnetic field $\gamma_{i j}^{0}$ acquires a complex phase expressed as an integral along the bond direction $\boldsymbol{d}_{i j}=\boldsymbol{r}_{j}-\boldsymbol{r}_{i}$.

With the CNTs oriented parallel to the $z$ axis, it is advantageous to choose a gauge in such a way that $\boldsymbol{A}_{\boldsymbol{B}}$ is independent of $r_{z}$. This is provided, e.g., by

$$
\boldsymbol{A}_{\boldsymbol{B}}(\boldsymbol{r})=\left(0, r_{x} B_{\|}, r_{y} B_{\perp}\right)
$$

giving a magnetic field $\boldsymbol{B}=\left(B_{\perp}, 0, B_{\|}\right)$with known components perpendicular and parallel to the tube axis. Throughout this work fields will be consider either perpendicular $\left(B_{\|}\right.$ $=0)$ or parallel $\left(B_{\perp}=0\right)$ to the tube axis. Arbitrary angles are of course possible as well, showing the expected crossover of both regimes.

Having chosen a linear gauge further simplifies the integration in Eq. (2) to a product:

$$
\gamma_{i j}=\gamma_{i j}^{0} \exp \left[\mathrm{i} \frac{2 \pi}{\Phi_{0}} \boldsymbol{d}_{i j} \cdot \boldsymbol{A}_{\boldsymbol{B}}\left(\frac{\boldsymbol{r}_{j}+\boldsymbol{r}_{i}}{2}\right)\right] .
$$

In the presence of a perpendicular magnetic field, it is thus necessary to consider the exact coordinates of the molecular structure at hand rather than-as sufficient for parallel or vanishing magnetic fields-their simple topological connectivity.

Density of states. For such an $r_{z}$-independent gauge field, the Hamiltonian of any quasi-1D periodic structure like a CNT stays periodic in the presence of a magnetic field. This allows the use of the Bloch theorem to derive the corresponding band structure. As can be seen in Fig. 3, the band structure is in general strongly distorted by an applied magnetic field. The density of states (DOS) can be determined from the magnetic band structure $E_{b}(k, \boldsymbol{B})$ via 


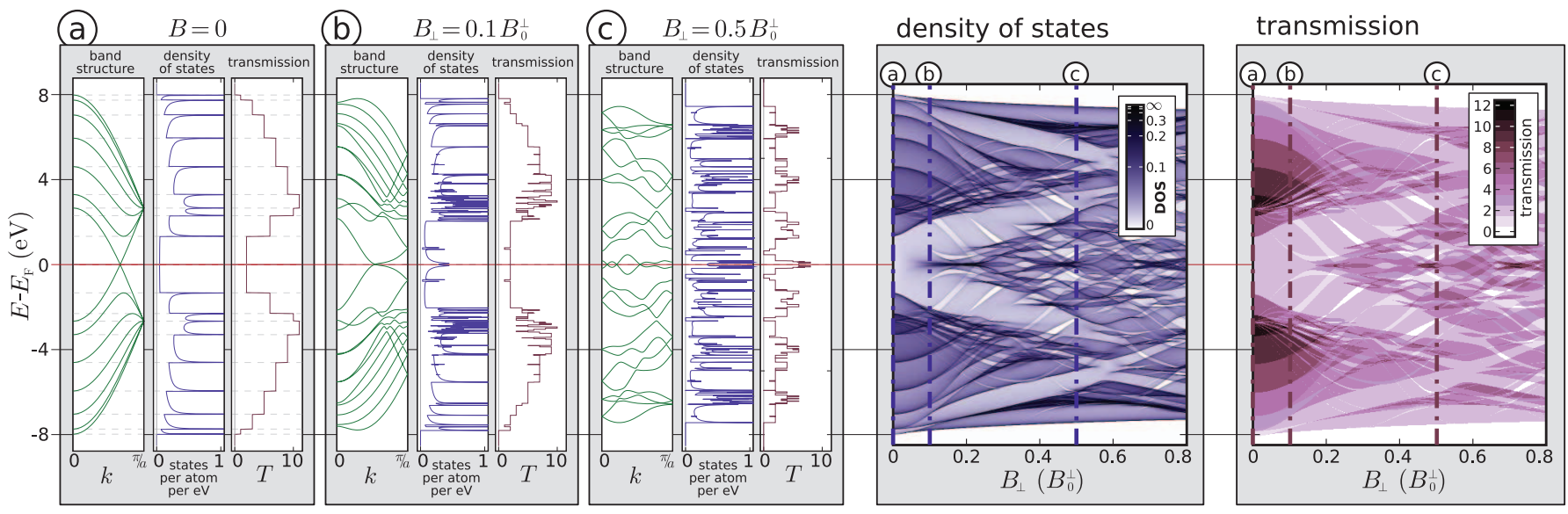

FIG. 3. (Color online) Scheme to illustrate the physical meaning of the butterfly plots. An external magnetic field distorts the band structure of a CNT in an intricate way. For any fixed magnetic field, the DOS and the transmission can be obtained directly from the band structure. (a), (b), and (c) are sections of the two right panels of the DOS and transmission vs $E$ and $B_{\perp}$.

$$
\rho_{\mathrm{DOS}}(E, \boldsymbol{B})=\frac{a}{2 \pi N_{b}} \sum_{b=1}^{N_{\mathrm{b}}} \int_{-\pi / a}^{\pi / a} d k \delta\left(E-E_{b}(k, \boldsymbol{B})\right)
$$

where $b$ is the band index and $a=3 d_{\mathrm{CC}} \sqrt{m^{2}+n^{2}+m n}$ / $\operatorname{gcd}(3 n, n-m)$ the length of the unit cell of an $(n, m)$ CNT. $E_{b}(k, \boldsymbol{B})$ is obtained by direct diagonalization of the CNT Hamiltonian via the Bloch ansatz (see Appendix A). Since we work in a basis of one orbital per atom, the number of bands $N_{\mathrm{b}}$ equals the number of atoms in the unit cell $N$ $=4\left(n^{2}+m^{2}+n m\right) / \operatorname{gcd}(3 n, n-m)$, growing with the diameter and dependent on the helicity angle (deviation from the armchair or zigzag configuration). The plot of the DOS directly reflects the distortions of the band structure caused by the magnetic field.

Alternatively, Green-function-based approaches allow one to resolve the DOS within different atoms in the same unit cell by introducing the local density of states

$$
\rho_{\mathrm{LDOS}_{\boldsymbol{r}_{i}}}(E, \boldsymbol{B})=-\frac{1}{\pi} \operatorname{Im} \mathcal{G}_{\boldsymbol{r}_{i}, \boldsymbol{r}_{i}}(E, \boldsymbol{B}),
$$

where $\mathcal{G}_{\boldsymbol{r}_{i}, \boldsymbol{r}_{i}}$ is the space-diagonal component of the lattice Green function matrix (see Appendix B)

$$
\mathcal{G}(E, \boldsymbol{B})=\left[E-\mathcal{H}(\boldsymbol{B})+\mathrm{i} 0^{+}\right]^{-1} .
$$

Of course by tracing the LDOS within the different atoms of the same unit cell, one can restore the full DOS as $\rho_{\mathrm{DOS}}(E, \boldsymbol{B})=\frac{1}{N} \sum_{\boldsymbol{r}_{i}} \rho_{\mathrm{LDOS}_{\boldsymbol{r}_{i}}}(E, \boldsymbol{B})$.

Butterfly plots. To capture the continuous evolution of the band structure with growing magnetic fields, it is very convenient to visualize the DOS in butterfly plots, as illustrated in Fig. 3. The resemblance to the well-known Hofstadter butterfly of 2D lattice electrons ${ }^{1}$ becomes very clear for CNTs of large diameter (see Fig. 7 below). A common feature to butterfly plots of all quasi-1D systems are the pronounced band edges, caused by van Hove singularities in the DOS..$^{35}$
In Fig. 1, a $(6,6)$ CNT Hofstadter butterfly is plotted as a reference for further comparisons. For the parallel field, the behavior is perfectly periodic for integer multiples of the flux quantum $\Phi_{0}=h / e$ penetrating the tube cross section $r^{2} \pi$. Starting as a metallic CNT at $B=0$, the gap opens and closes periodically. ${ }^{11}$

For perpendicular fields with their natural scale of one flux quantum per graphene plaquette, the overall behavior is not periodic. This can be understood due to the presence of plaquettes at various angles toward the field, capturing different, in general incommensurate, fractions of the flux quantum. However, a number of features from the underlying graphene structure are still visible at the diameterindependent scale of $B_{0}^{\perp}$.

Important to note is the difference in the behavior for small fields: while the parallel field causes a linear Zeeman split of the states with opposite angular momentum, small perpendicular fields generally cause quadratic energy shifts.

All plots are of course symmetric in the magnetic field sign, which is why only half butterfly plots are shown. Different is the case of the $E \rightarrow-E$ symmetry which is related to the particle-hole symmetry. The latter is present in the $\pi$-orbital description of SWCNTs but is broken by the interwall interaction in DWCNTs.

Transport observables in quasi-1D systems. As can also be seen in Fig. 3, it is straightforward to apply the same scheme not only to the density of states, but just as well to other properties like the quantum mechanical transmission $T$ of a quasi-1D system. The latter is the dimensionless zerotemperature conductance after the Landauer theory of phasecoherent transport: ${ }^{36} G=G_{\mathrm{K}} T$, where $G_{\mathrm{K}}=2 e^{2} / h$ is the conductance quantum and inverse of the von Klitzing resistance. The calculation of the transmission, which involves a renormalization procedure for the semi-infinite carbon nanotube leads $^{37}$ by means of the energy-dependent injection rates $\Gamma_{\mathrm{L} / \mathrm{R}}$ and the Green function $\widetilde{\mathcal{G}}$ projected on a finite nanotube partition, can be cast into the Fisher and Lee formula ${ }^{38}$

$$
G=\frac{2 e^{2}}{h} \operatorname{Tr}\left\{\Gamma_{\mathrm{L}} \widetilde{\mathcal{G}} \Gamma_{\mathrm{R}} \widetilde{\mathcal{G}}^{\dagger}\right\}
$$



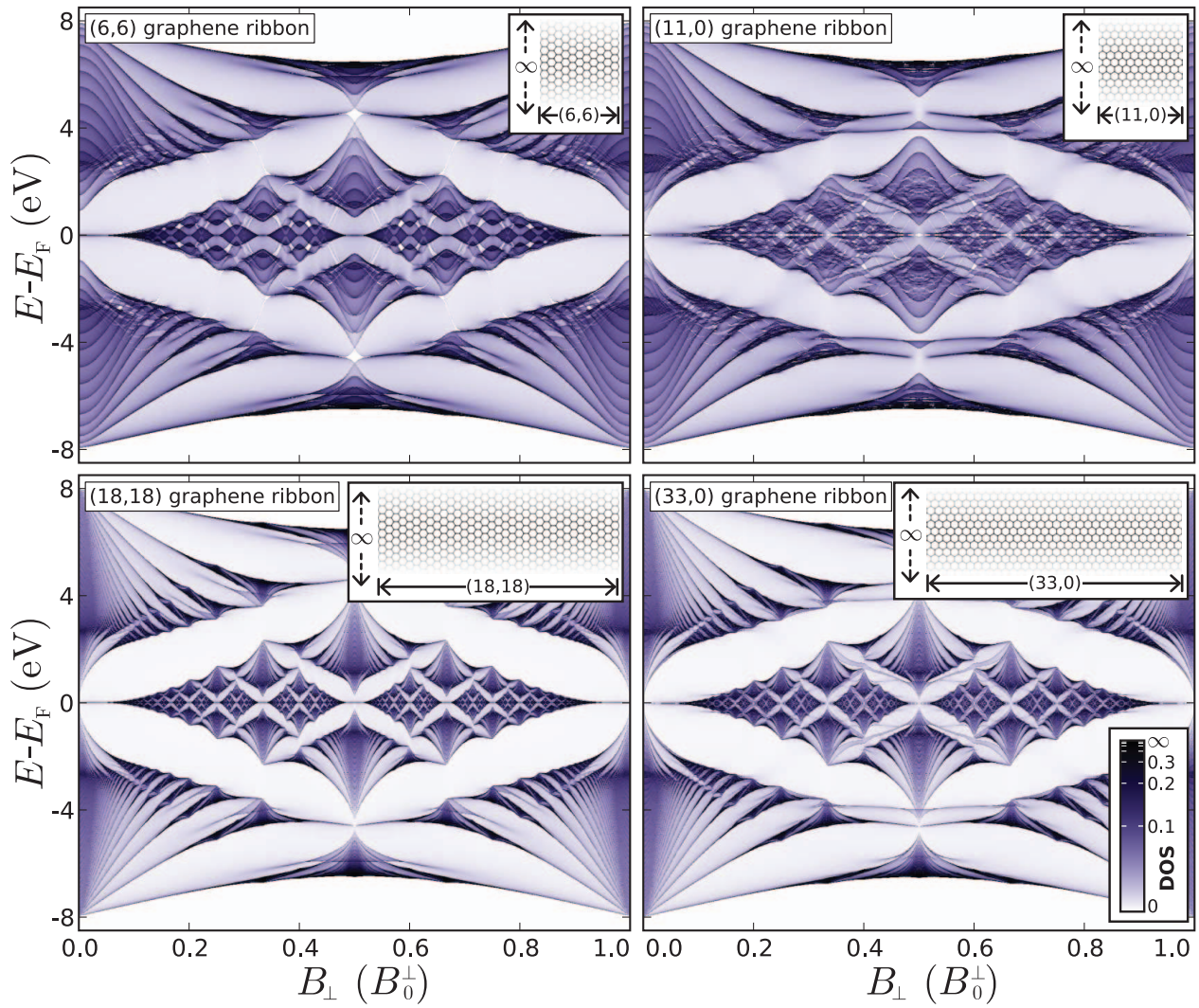

FIG. 4. (Color online) DOS in a graphene ribbons of infinite length and various widths and internal orientations, pierced perpendicularly by magnetic fields. Each ribbon can be classified as an unrolled CNT: The "chiral" vectors refer to the SWCNT which, when unrolled, would result in the corresponding planar ribbon. The density of states is normalized to the number of atoms per unit cell to give a comparable visual appearance.
Still, for a periodic structure-as is the case for the systems at hand-the quantum mechanical transmission is simply a band-counting algorithm, and as such contains less information than the band structure itself or the DOS. This is very different from magnetotransport through finite CNTs: Scattering at the contacts leads to resonant tunneling, resulting in spectroscopy of the electronic states of the finite tube. ${ }^{39,40}$ This spectrum may show strong dependence on magnetic fields, even in regions of flat bands, ${ }^{16}$ resulting in quantum-dot-like physics. $^{41}$

Relation to $2 D$ periodic structures. It is important to note some similarities, but also some fundamental differences between the butterfly plots of quasi-1D structures and those in the original work by Hofstadter ${ }^{1}$ and later generalizations ${ }^{24,25}$ which handled 2D periodic structures. Starting out from an analogous Hamiltonian and also using the Peierls substitution to implement the magnetic field, the most striking difference is that, for a $2 \mathrm{D}$ periodic structure, it is not possible in general to choose a gauge in such a way that the resulting Hamiltonian has the same translational symmetry as the underlying system. For rational values of the magnetic flux per unit cell, one can still find a larger effective unit cell but, for irrational values, this is not possible at all, which ultimately leads to the fractal structure of the energy spectrum found by Hofstadter, similar to that displayed in the lower panel of Fig. 7. In contrast, the quasi-1D structure of CNTs results in a fixed number of bands, leading to a pseudofractal spectrum, with the recursion of self-similarity limited by the transverse length cutoff of the system.

Graphene ribbons. Since the recent experimental success in isolating single sheets of graphene, ${ }^{42,43}$ the exotic Diraclike electronic structure has become the focus of several studies. Epitaxially grown graphene has been used to laterally confine electrons and determine coherence lengths studying weak-localization effects in magnetotransport measurements. ${ }^{44}$ For understanding the relation between the butterfly of a 2D graphene sheet and these quasi-1D carbon nanotubes, it is instructive to take a look at graphene ribbons as an intermediate step. An $(n, m)$ graphene ribbon is simply a planar "unrolled" $(n, m) \mathrm{CNT}$, periodic in one dimension and finite in the other. As in the original Hofstadter butterfly, ${ }^{1}$ the ribbon butterfly plots are periodic as a function of the perpendicular magnetic field due to the equal flux piercing any hexagonal plaquette forming the honeycomb lattice (see Fig. 4). As the ribbon width increases the butterfly plots tend to the Hofstadter butterfly of a graphene layer as visible in the bottom panel of Fig. 7 .

Cusp catastrophes. One striking detail ubiquitous in butterfly plots are the cusp catastrophes appearing at specific positions of energy and magnetic fields (see Fig. 5). These are points where, with changing magnetic field, some band is continuously deformed from a strictly monotonic curve into a band with two adjacent zero-group-velocity points. At the exact point where this mathematical catastrophe happens, both the first and the second derivatives of $E(k)$ are zero. A wave packet of this energy and momentum will have both its velocity and its spreading suppressed leading to a special kind of localization not unlike that of Landau levels.

\section{SINGLE-WALL CARBON NANOTUBES}

For SWCNTs we consider only the radial $p$ orbitalsforming the most electronically relevant $\pi$ bands-and only 


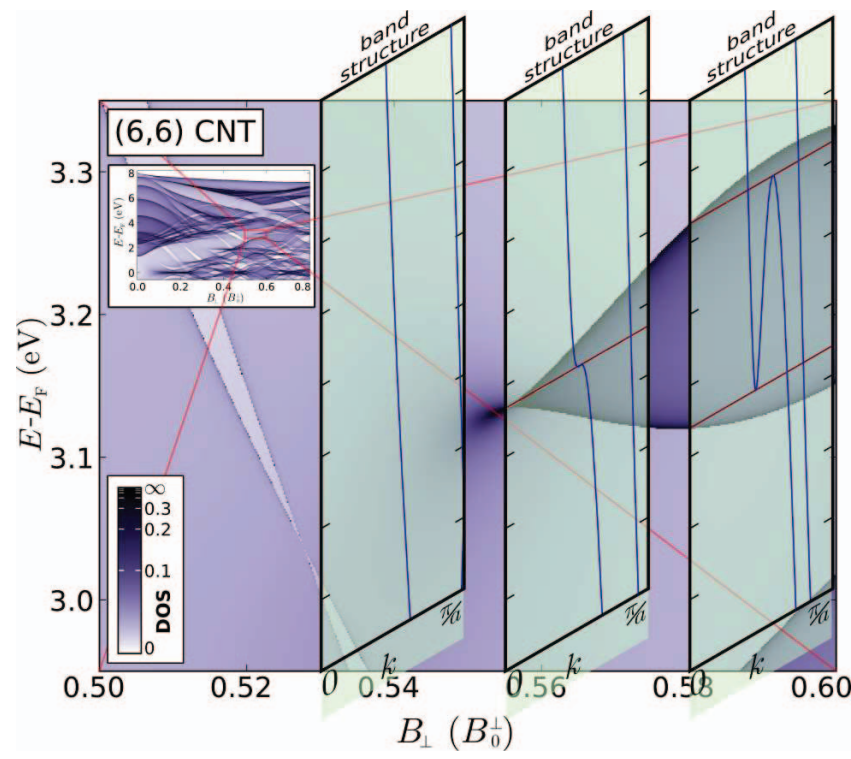

FIG. 5. (Color online) Cusp catastrophes are ubiquitous in butterfly plots. The band structure at magnetic fields below, at, and above the critical magnetic field shows the smooth transition from a strictly monotone band into a third-order parabolic band with changing magnetic field.

interactions between nearest neighbors, setting $\gamma_{i j}^{0}=\gamma_{0}$ $=2.66 \mathrm{eV}$. This has been shown to be an excellent approximation in explaining electronic structural and transport properties of SWCNTs. ${ }^{5-7}$ The on-site energy $\varepsilon_{i}=\varepsilon_{0}$ is constant for all atoms and defines the Fermi energy $E_{\mathrm{F}}=\varepsilon_{0}$ of a neutral CNT. Ignoring an offset in the energy, we can simply choose $\varepsilon_{0}=0$. Zeeman splitting could also easily be included in this calculation as $\varepsilon_{0}= \pm g \mu_{\mathrm{B}} B / 2$ and would result visually in an overlay of two butterfly plots sheared against each other linearly with growing magnetic fields. The intensity of this effect at the critical plaquette field scale is $g \mu_{\mathrm{B}} B_{\text {plaquette }}$ $=9.1 \mathrm{eV}$.

The special case of parallel magnetic fields: Shortcut via the zone-folding method. As an alternative to calculating the electronic bands of a SWCNT via the procedure described above, one could calculate the spectrum of graphene and then apply periodic boundary conditions in the angular direction of the CNT (zone folding). For magnetic fields parallel to the tube axis, this method is still applicable: the phase gathered by an electron moving on a closed loop around the tube circumference can simply be included in the boundary conditions. This results in a shift of the allowed discretized quasimomenta in the reciprocal space. For perpendicular magnetic fields, however, this method breaks down and one has to consider the full geometry of the CNT.

\section{A. Structural properties}

Chirality dependence. Several features can be found when comparing the magnetic spectra of tubes with different chiralities though similar diameter (see Figs. 1 and 6). (i) The behavior of the gap around the charge neutrality point $E$ $=E_{\mathrm{F}}$ is very helicity dependent: a parallel magnetic field always opens and closes the gap periodically as a consequence
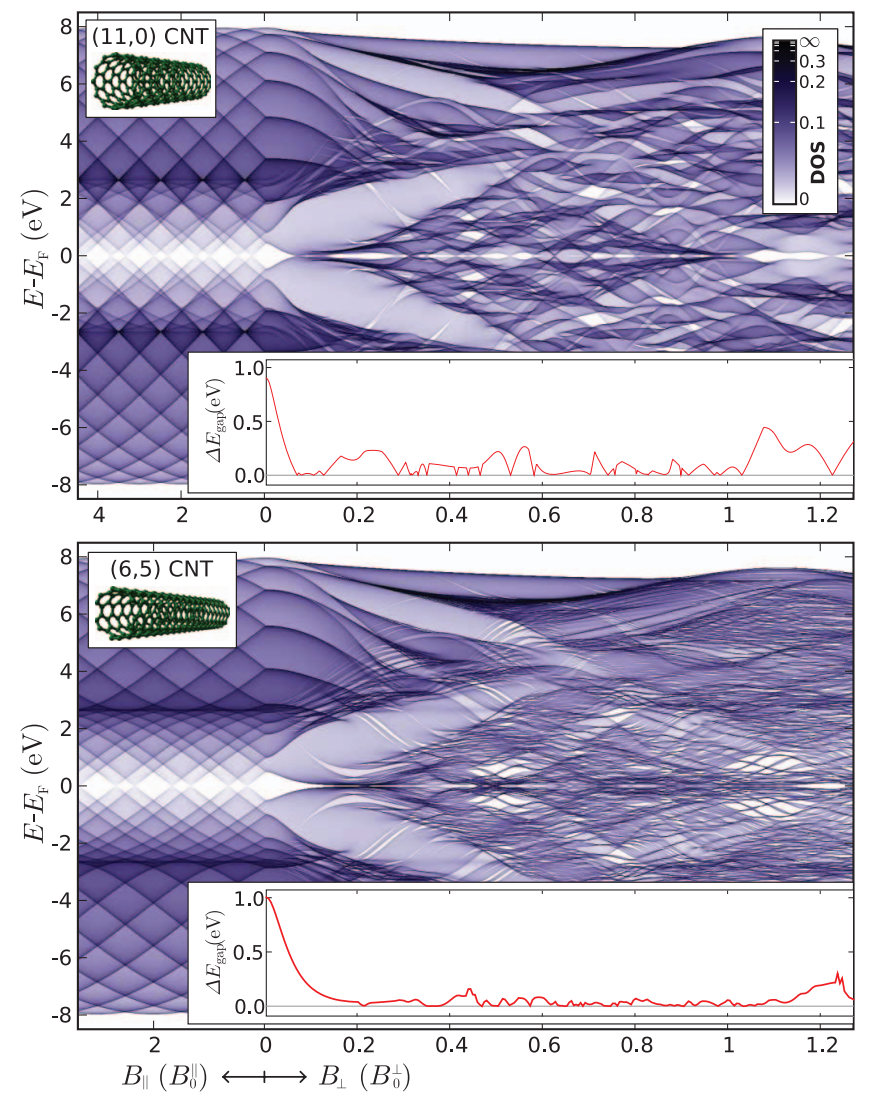

FIG. 6. (Color online) Two semiconducting SWCNTs of similar diameter as the $(6,6) \mathrm{CNT}$ in Fig. 1. The band gap oscillates irregularly with increasing perpendicular field. The large unit cell of the the chiral $(6,5)$ tube leads to bands with low dispersion as soon as the rotational symmetry is broken by the perpendicular magnetic field.

of the integer number of fluxes per nanotube cross section. This phenomenon is independent of whether the tube is metallic or semiconducting at $B=0$. In contrast, for perpendicular fields there are distinctions. Armchair CNTs stay strictly metallic for any perpendicular field, as can be understood from supersymmetry arguments. ${ }^{45}$ On the other hand, the gaps of the two semiconducting CNTs in Fig. 6 do open and close in an aperiodic, though oscillatory, pattern. The gap closes to zero in single points of specific values of $B_{\perp}$ and opens again. Closer observations of a larger set of CNTs reveal that this also happens for semimetallic tubes like the $(3 n, 0)$ zigzag CNTs. The precise opening and closing pattern carries an intrinsic complexity; its statistical behavior, however, seems to depend mostly on the number of atoms in the unit cell. (ii) Another general effect of the large unit cell in the $(6,5) \mathrm{CNT}$ is that in this chiral tube with its large number of plaquettes at different angles towards the field, the high symmetry of the original system is broken down very efficiently by the magnetic field, resulting in a larger number of bands of very low dispersion. The magnetic field effectively localizes the electrons in nonpropagating Landau-like states.

Diameter dependence. Figure 7 shows the evolution toward the graphene Hofstadter butterfly of the magnetic spectrum of armchair CNTs as a function of their diameter. The $(200,200)$ SWCNT has a diameter of $27 \mathrm{~nm}$, comparable to 

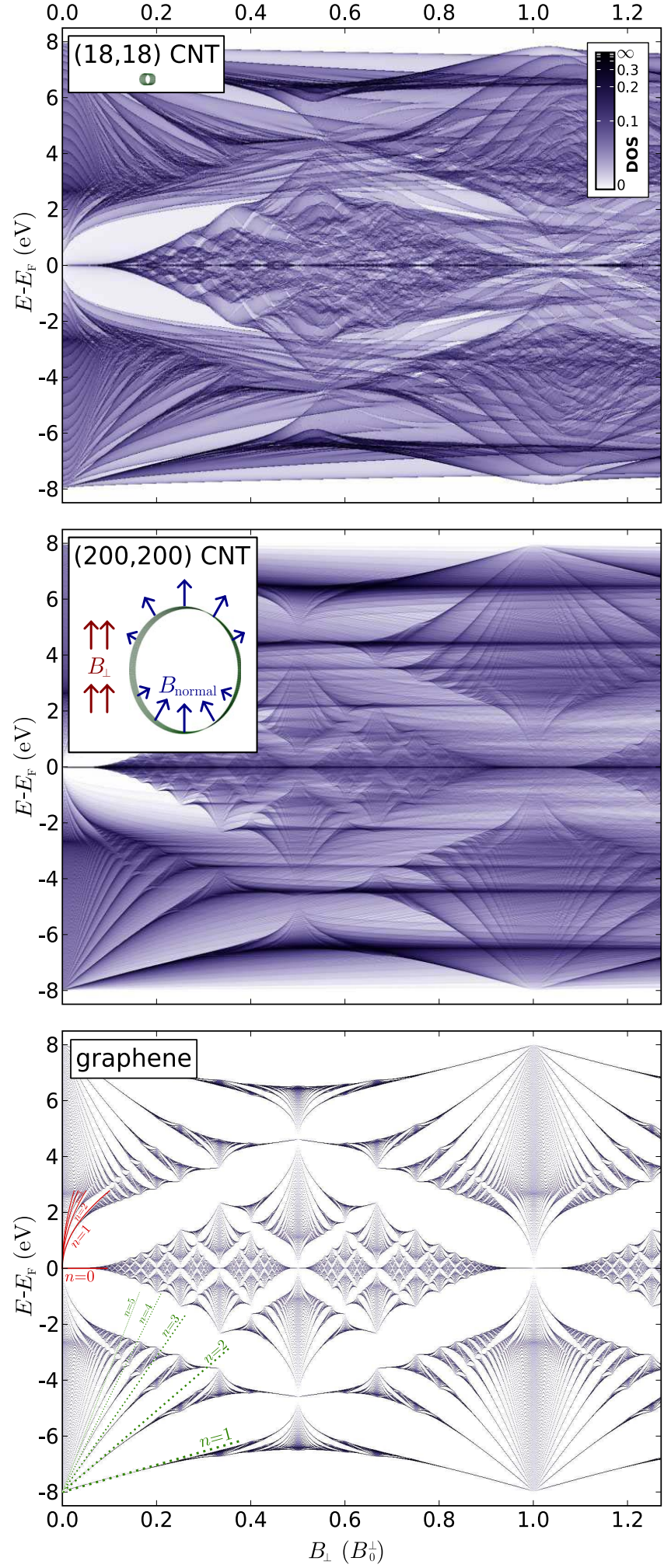

FIG. 7. (Color online) Comparing different diameters: the large $(200,200)$ CNT bears strong resemblance to the Hofstadter butterfly of graphene (Ref. 25) combined with the curvature effects (details in text). The straight lines at the lower left corner of the graphene butterfly (bottom panel) indicate the Landau states obtained from an effective mass continuum theory [see Eq. (8)]. The parabolic lines near $E_{\mathrm{F}}$ in the same plot indicate the relativistic Landau levels obtained from the Dirac-like dispersion of graphene [see Eq. (9)]. the external shell of a typical MWCNT, and is thus of great interest. The overlaid visual effect-resembling watercolors "flowing" toward the right hand side-can also be understood by a simple picture. For large enough diameters, the CNT consists of regions of nearly flat graphene, each at a different angle toward the magnetic field, thereby experiencing a different normal component of the magnetic field, as visible in the inset of the middle panel of Fig. 7. Since the DOS is an average over the LDOSs at the different unit cell atoms, one ends up with a sum of different graphene Hofstadter butterflies, stretched to different effective fields, the stretch being minimal where the magnetic field is normal to the tube wall and maximal where it is tangential. Overlaying these differently stretched graphene butterflies results in the "flowing" appearance of the butterfly of large diameter tubes.

At the lower and upper energy edges, one can clearly see the emergence of linear Landau levels and the characteristic fractal structure of the graphene butterfly is unmistakenly visible at the same scale of the magnetic field. In fact near the top and bottom of the graphene $\pi$ energy band (of width $2 W=6 \gamma=16 \mathrm{eV})$, electrons have an effective mass of $m^{*}$ $=2 \hbar^{2} / 3 \gamma d_{\mathrm{CC}}^{2} \approx 0.95 m_{e}$, leading to a cyclotron frequency $\omega\left(B_{\perp}\right)=e B_{\perp} / m^{*}$, so one could write

$$
E=\mp W \pm \hbar \omega(B)\left(n+\frac{1}{2}\right)
$$

with $n=0,1,2, \ldots$, which fits nicely with the numerical results (as indicated by the straight lines in the bottom panel of Fig. 7).

Around the Fermi energy, the Dirac-like dispersion of graphene leads to the so-called relativistic Landau levels, ${ }^{46,47}$ following

$$
E= \pm v_{\mathrm{F}} \sqrt{2 n \hbar e B_{\perp}}
$$

with $n=0,1,2, \ldots$ and the Fermi velocity $v_{\mathrm{F}}=3 \gamma d_{\mathrm{CC}} / 2 \hbar$. These levels, which can be clearly observed in the Hofstadter butterfly of graphene (left edge in the bottom panel of Fig. 7 ), are also responsible for the recently observed anomalous quantum Hall effect of graphene. ${ }^{42,43}$

Snake states. The view of the total DOS of a large SWCNT as the sum of different contributions from the regions at various angles around the tube circumference can be confirmed by taking a look at the LDOS at individual atom positions. As can be seen in Fig. 8, the LDOS at $\theta=0$, where the magnetic field pierces the wall perpendicularly, resembles very much the butterfly of the planar graphene sheet. The electrons here show very low dispersion, similar to Landau levels. At $\theta=\pi / 2$, on the other hand, the magnetic field is tangential to the CNT wall and therefore has far less effect on the electron dispersion. An understanding of the electronic states in these regions can be gained by considering classical electrons confined to the surface of a cylinder: As the effective magnetic field (the projection of the field onto the tube normal) changes sign at $\theta=\pi / 2$, the curvature of an electron trajectory will also switch orientation each time the electron crosses this "equator" line, leading to a snakelike movement of the electron. ${ }^{45,48}$ 

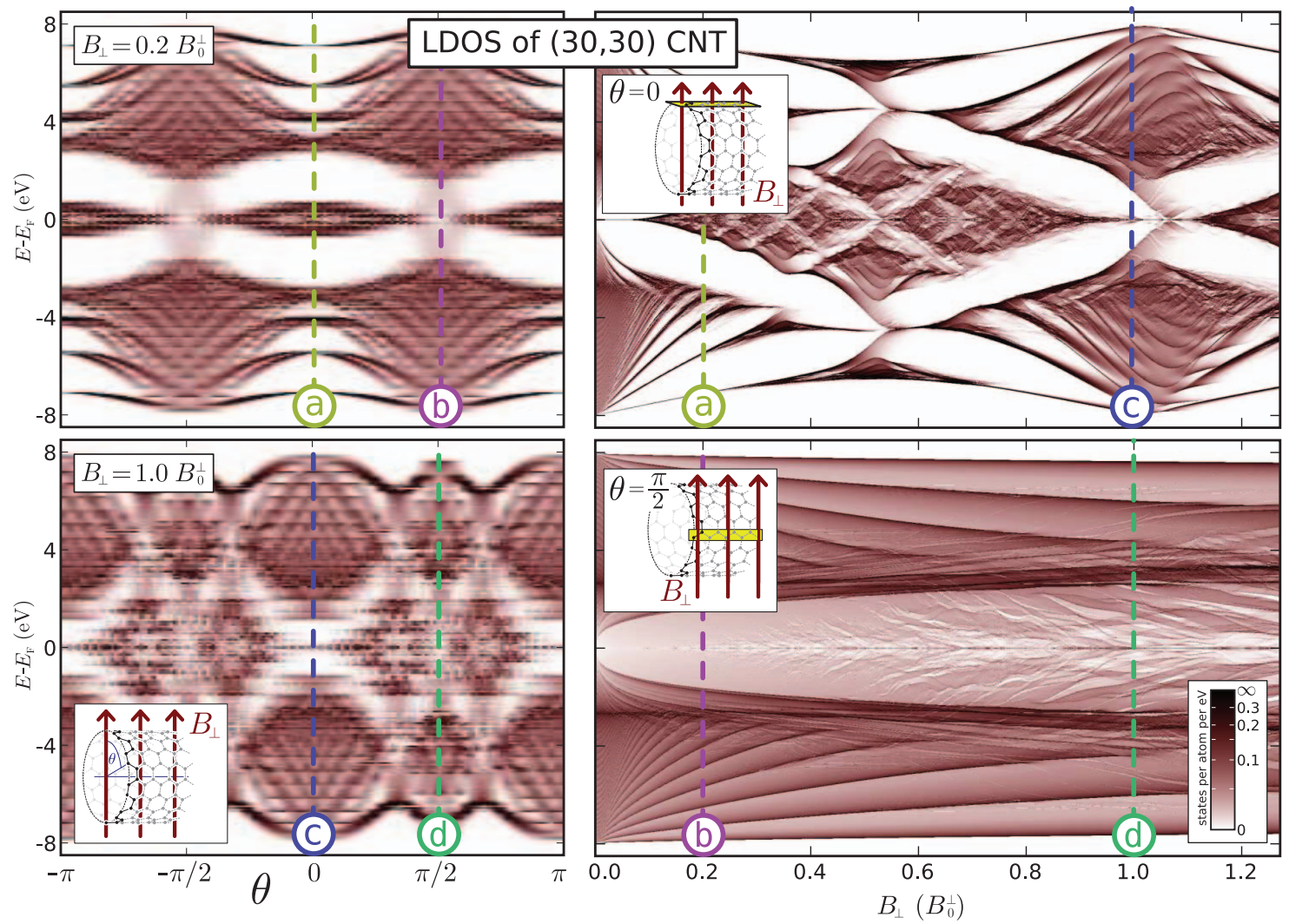

FIG. 8. (Color online) Decomposition of the density of states into the contributions of particular atoms (identified by their angle $\Phi$ toward the magnetic field direction). A plaquette at angle $\theta$ captures a flux of $B_{\perp} A_{\text {plaquette }} \cos \theta$. The region at $\Phi=0$ experiences a perpendicular field piercing the tube wall, very much as in the plain graphene sheet (Fig. 7). The regions at $\Phi=\pi / 2$ experiences a field tangential to the tube wall, leading to a much smaller flux per plaquette, resulting in a stretched impression of the butterfly. The DOS butterfly over the whole CNT unit cell is an overlay of these and many intermediate pictures. In the angle-resolved plot for $B=0.2 B_{0}^{\perp}$ one can see a smooth transition between a region with Landau levels and a region with normal band dispersion. For the stronger field, the systems goes through two oscillations along the angle.

\section{B. Analytical model}

In order to shed some light of intuition on our results, we may consider the physics of a structureless hollow cylinder, a tubule, in a perpendicular magnetic field. (Similar systems in parallel magnetic fields have been studied before. ${ }^{49,50}$ ) This system bears some similarity to a "Hall bar", with the crucial difference that it does not have borders that could carry edge states. Instead, it has two flanks where the magnetic field is tangential to the tube and therefore the radial component of the magnetic field-which is the effective field experienced by electrons confined to the cylinder surface-vanishes. To understand where charges do accumulate, we consider this system in cylindrical coordinates $(\theta, z)$ at fixed radius $r$. By Eq. (3), a perpendicular magnetic field leads to a gauge field

$$
\boldsymbol{A}(\theta)=B_{\perp} r \sin \theta \boldsymbol{e}_{z}
$$

in cylindrical coordinates. With this, the Hamiltonian of an electron restricted to the tube surface becomes

$$
\mathcal{H}=\frac{1}{2 m r^{2}} p_{\theta}^{2}+\frac{1}{2 m}\left(p_{z}-e B_{\perp} r \sin \theta\right)^{2}
$$

which can be viewed as that of an electron in $2 \mathrm{D}$ with periodic boundaries in a nonuniform magnetic field. ${ }^{51,52} \mathrm{~A}$ simi- lar system-a $2 \mathrm{D}$ strip ranging over $[-L / 2, L / 2]$ in the $y$ direction and infinite in the $z$ direction, placed in a linearly varying magnetic field $\boldsymbol{B}=B_{0} y \boldsymbol{e}_{x}$-was first studied in 1992 by Müller, ${ }^{48}$ who identified two new classes of states: one at finite magnetic field propagating perpendicularly to the field gradient direction with looping trajectory and low velocity, the other around the line $B=0$, propagating in the opposite direction at higher velocity with a snakelike trajectory. To solve our system, we can exploit the $z$ invariance and do an ansatz for the wave function: $\Psi(\theta, z)=\psi_{k_{z}}(\theta) e^{i k_{z} z}$. Our problem reduces to that of a particle in one dimension with a $k_{z}$-dependent potential:

$$
\mathcal{H}_{k_{z}}=\frac{1}{2 m r^{2}} p_{\theta}^{2}+V_{k_{z}}(\theta)
$$

$$
V_{k_{z}}(\theta)=\frac{1}{2 m}\left(\hbar k_{z}-e B_{\perp} r \sin \theta\right)^{2} .
$$

For $\left|\hbar k_{z}\right|<\left|e B_{\perp} r\right|$, this potential has two minima at $\theta_{\text {min }}$ $=\pi / 2 \pm \arccos \left(\hbar k_{z} / e B_{\perp} r\right)$. A harmonic approximation at either of these minima yields the approximate Hamiltonian 

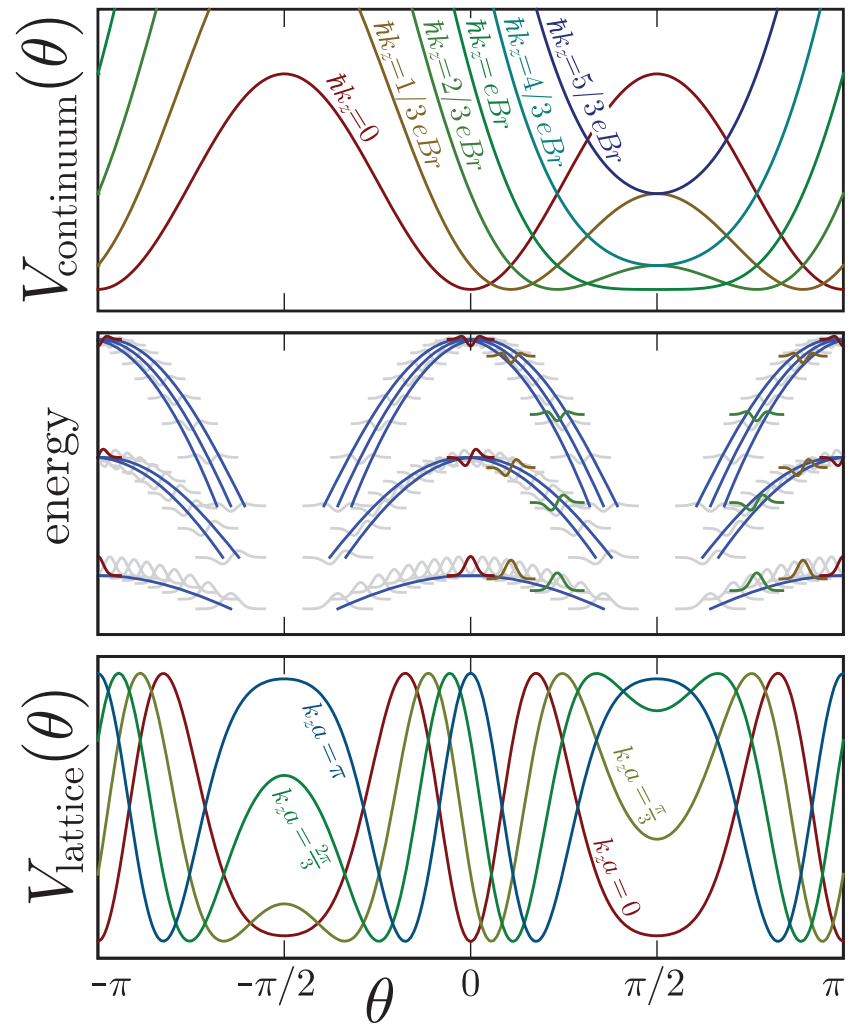

FIG. 9. (Color online) Analytical solution of the continuum model. Top panel: the $k_{z}$-dependent effective potential of free electrons confined to a continuum cylinder in a magnetic field perpendicular to the tube. Middle panel: first three eigenstates of the harmonic approximation to the above potential for various $k_{z}$ (highlighted wave functions correspond to the selected potentials in the first panel). Each wave function is shifted to the corresponding energy. Superimposed are the lines followed by the extrema of the wave functions. The same pattern can be found in the top left panel of Fig. 8, where the maxima of the DOS show the maxima of the various energy eigenstates. Bottom panel: The $k_{z}$-dependent effective potential of a discretized tube showing a large number of minima. States located in narrow minima have higher energy, so the low-energy spectrum is mainly determined by the widest potential minima.

$$
\mathcal{H}_{k_{z}}=\frac{p_{\theta}^{2}}{2 m r^{2}}+\frac{1}{2 m}\left[\left(e B_{\perp} r\right)^{2}-\left(\hbar k_{z}\right)^{2}\right]\left(\theta-\theta_{\text {min }}\right)^{2}
$$

with the spectrum

$$
E_{n}\left(k_{z}\right)=\frac{\hbar}{m r} \sqrt{\left(e B_{\perp} r\right)^{2}-\left(\hbar k_{z}\right)^{2}}(n+1 / 2) .
$$

From this dispersion relation, we can directly retrieve the group velocity

$$
v_{n}\left(k_{z}\right)=-\frac{\hbar}{m r} \frac{(n+1 / 2) \hbar k_{z}}{\sqrt{\left(e B_{\perp} r\right)^{2}-\left(\hbar k_{z}\right)^{2}}} .
$$

The wave functions in the harmonic potential are located around the minima $\theta_{\text {min }}$, so for low energies we can say in reverse that at each angle $\theta$ we find predominantly electrons with the longitudinal wave vector $k_{z}(\theta)=\left(e B_{\perp} r / \hbar\right) \sin \theta$.
Placing this into Eq. (11), we can retrieve an expression for the velocity of electrons moving at certain angles:

$$
v_{n}(\theta)=-\frac{\hbar}{m r}(n+1 / 2) \tan \theta .
$$

Now, the divergency at $\theta= \pm \pi / 2$ originates from the fact that the harmonic approximation breaks down when the two minima of $V_{k_{z}}(\theta)$ meet at this angle. Apart from this, however, one can see clearly the angular separation of electrons moving in both directions and the localization in Landau-like states at $\theta=0$ and $\theta=\pi$, where the magnetic field pierces the tube wall normally (see Fig. 9).

Such a continuum model can only be expected to hold for the CNTs at low magnetic fields with $B_{\perp} A_{\text {plaquette }} \ll \Phi_{0}$. As it turns out, some of the effects visible at higher fields can be understood qualitatively by studying a model of intermediate complexity: a square lattice cylindrical tube of lattice constant $a$. Coming from the continuum model and following Ref. 53, we can replace the continuous coordinates by integer indices: $(a m, a n):=(r \theta, z)$. Using a tight-binding model with on-site energy $\varepsilon_{0}$ and hopping parameter $\gamma_{0}$, the Hamiltonian acts on a wave function in the following way:

$$
\begin{aligned}
\mathcal{H} \Psi_{(m, n)}= & \varepsilon_{0} \Psi_{(m, n)}-\gamma_{0}\left(\Psi_{(m-1, n)}+\Psi_{(m+1, n)}\right) \\
& -\gamma_{0}\left(e^{-\mathrm{i} \varphi(m)} \Psi_{(m, n-1)}+e^{\mathrm{i} \varphi(m)} \Psi_{(m, n+1)}\right)
\end{aligned}
$$

where the phase factor $\varphi(m)=\frac{2 \pi a r B_{\perp}}{\Phi_{0}} \sin \frac{m a}{r}$ originates from the Peierls substitution Eq. (2). As in the continuum, the invariance in the $z$ direction can be exploited, now using a Bloch ansatz due to the discreteness of the system:

$$
\Psi_{(m, n)}=e^{\mathrm{i} k_{z} a n} \psi_{m}
$$

This leads to a finite Hamiltonian for any fixed $k_{z}$ $\in[-\pi / a, \pi / a)$ :

$$
\begin{gathered}
\mathcal{H}_{k_{z}} \psi_{m}=-\gamma_{0}\left(\psi_{m-1}+\psi_{m+1}\right)+V_{k_{z}}(m) \psi_{m}, \\
V_{k_{z}}(m)=\varepsilon_{0}-2 \gamma_{0} \cos \left(k_{z} a-2 \pi \frac{r a B_{\perp}}{\Phi_{0}} \sin \frac{m a}{r}\right) .
\end{gathered}
$$

The most significant difference to the effective potential of the continuum model Eq. (10) is the replacement of the square law by a cosine one. This has the effect that the potential does not grow indefinitely for large magnetic fields, but instead oscillates, forming several minima at various angles $\theta$, as seen in Fig. 9. In combination with the discretization of the angle, this potential leads to the formation of a complex pattern in the angular dependence of the density of states, as it can be observed is the LDOS at high magnetic fields also displayed in Fig. 8.

To capture more details in a model, an appropriate step would be the implementation of the correct dispersion at the Fermi energy: The characteristic cones at the Fermi points of graphene can be approximated by a Dirac-like Hamiltonian. For a detailed study of the magnetic spectrum of Diracelectrons on a cylindrical surface, see Refs. 45 and 54. 

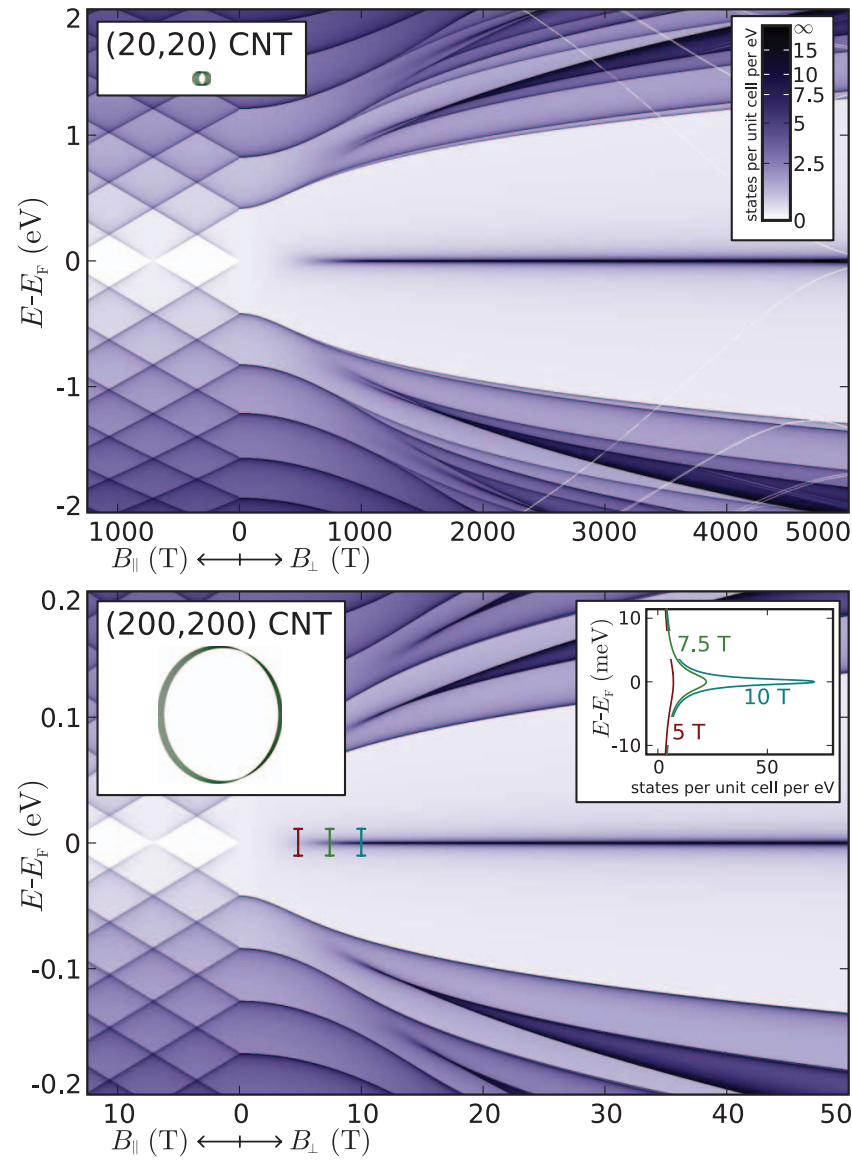

FIG. 10. (Color online) Zoom into the butterfly of two different armchair SWCNTs. The scales, including the color scale, are chosen according to the scaling law given in the text to produce comparable representations of the data. The tubes correspond to diameters of $2.7 \mathrm{~nm}$ (top) and $27 \mathrm{~nm}$ (bottom). The inset in the bottom panel illustrates the shape of the peak at the Fermi level. The white lines crossing the plot in the upper panel are caused by small avoided crossings in the band structure.

\section{Experimentally accessible perpendicular magnetic fields}

In recent experiments, optical transitions in CNTs were studied in magnetic fields up to $75 \mathrm{~T} .{ }^{55}$ New experiments are in preparation to go up to $200 \mathrm{~T}$ and even $2-3 \mathrm{kT}$. $^{56}$

In perpendicular fields of this magnitude, as displayed in Fig. 10, the first onset of the band structure distortions can be seen clearly in large CNTs, comparable with the outer shell of typical MWCNTs, measuring up to tens of nm in diameter.

In particular the plots show very clearly the rapidly changing van Hove singularities, resembling those of Fig. 1 for a $(6,6) \mathrm{CNT}$, but at much lower magnetic field scale. Moreover, the $E=E_{\mathrm{F}}$ graphene state, which is due to the peculiar distortion of the Dirac-like linear dispersion into a strongly nonlinear one, ${ }^{18}$ emerges at lower fields with increasing diameters.

Most notable is the scaling law that can be found in the butterfly plot of large tubes at low fields near the Fermi energy: For two different armchair CNTs with the chiral vectors $(m, m)$ and $\left(m^{\prime}, m^{\prime}\right)$ it can be expressed as

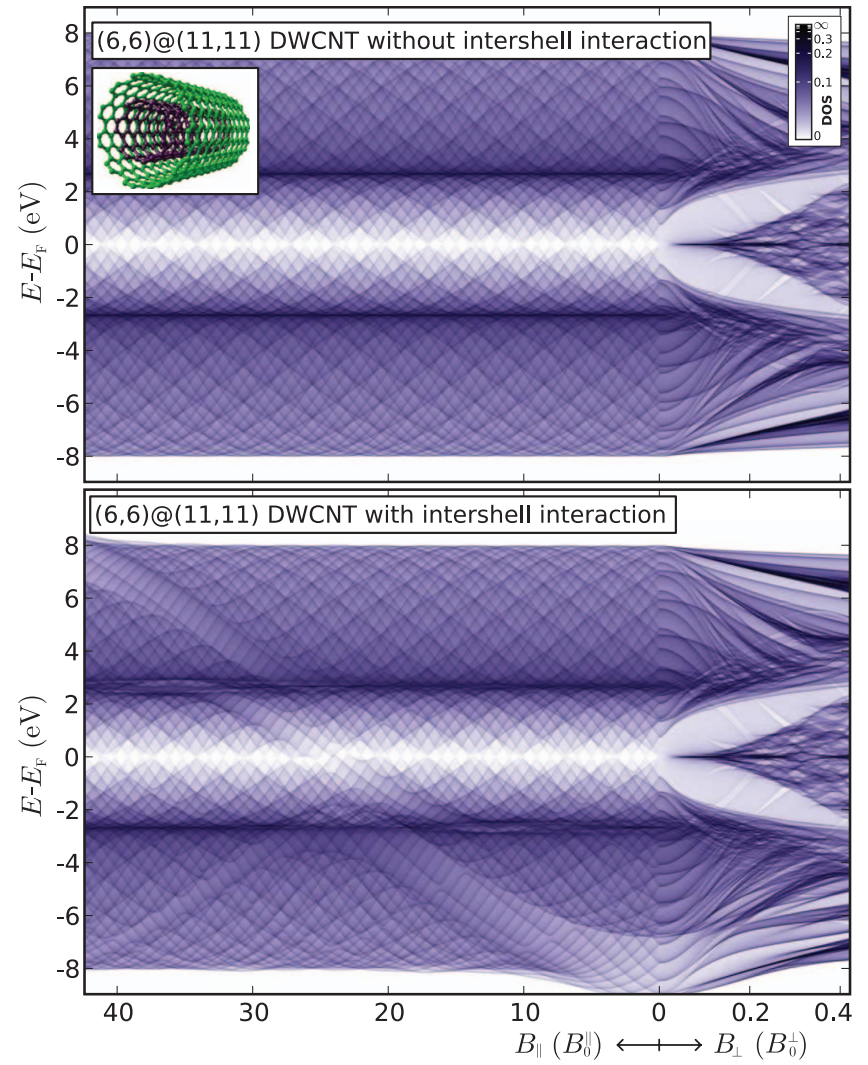

FIG. 11. (Color online) Butterfly plot of a $(6,6) @(11,11)$ doublewall CNT. In the upper panel, the interwall interaction is switched off, resulting in an overlay of the butterflies of two independent SWCNTs. In the lower panel, the interwall interaction gives rise to a number of new features (see text for details).

$$
\rho_{\operatorname{DOS}_{(m, m)}}(E, \boldsymbol{B})=\frac{m^{\prime}}{m} \rho_{\operatorname{DOS}_{\left(m^{\prime}, m^{\prime}\right)}}\left(\frac{m}{m^{\prime}} E, \frac{m^{2}}{m^{\prime 2}} \boldsymbol{B}\right) .
$$

This scaling is followed approximately already for small CNTs and becomes very precise for large diameters, converging toward a DOS that is reproducible from a model of Dirac electrons on a continuum cylinder. ${ }^{45}$ The peak at the Fermi energy also follows this scaling law. Within the region of scaling, the maximum of the peak at $E=E_{\mathrm{F}}$ grows exponentially with the magnetic field while its integral grows linearly.

It is important to note that the scaling is not an effect of the curvature, but of the discretization of the transversal momentum, since it can be observed in graphene ribbons as well.

\section{DOUBLE-WALL CARBON NANOTUBES}

While SWCNTs and MWCNTs have been studied intensely over the past 15 years, it has only recently become possible to produce DWCNTs of high purity and quality, ${ }^{57,58}$ fueling the interest in details about the interwall interaction. Previous studies have shown an interesting interplay between magnetic fields parallel to the DWCNT axis and the interwall interaction near the Fermi energy. ${ }^{59}$ A minimal Hamiltonian of a DWCNT can be set up as 


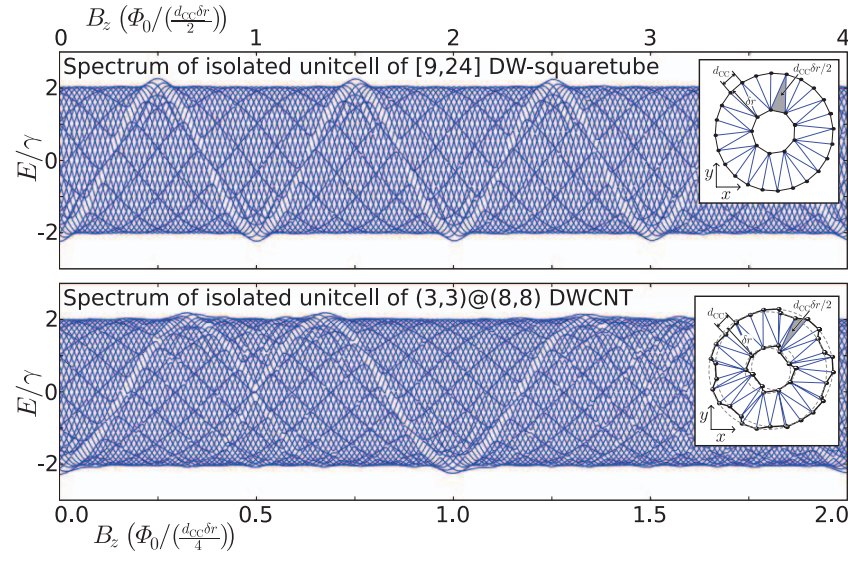

FIG. 12. (Color online) Upper panel: Spectrum of a system of two concentric atomic rings. Atom spacing and coupling inside each ring are taken from graphene. The distance $\delta r$ between the rings as well as the the parametrization of the coupling between the rings follow those given in the text for DWCNTs. The sketch displays the prevalent links between the shells. Even though the geometry is irregular, the area of circular paths is very near to integer multiples of $d_{\mathrm{CC}} \delta r / 2$, leading to a clear periodicity of the modulation in the spectrum. Lower panel: An isolated unit cell of a DWCNT with the same radii as the planar double ring of the upper panel. This system has smallest closed loops at an angle against the magnetic field, resulting in an effective smallest area of $d_{\mathrm{CC}} \delta r / 4$ and leading to a doubling of the period. Furthermore, the system has two atoms in the rotational periodic cell, leading to two interlaced modulations.

$$
\mathcal{H}=\sum_{\langle i, j\rangle} \gamma_{i j}(\boldsymbol{B}) c_{i}^{\dagger} c_{j}+\sum_{\langle\langle i, j\rangle\rangle} \tilde{\gamma}_{i j}(\boldsymbol{B}) c_{i}^{\dagger} c_{j}
$$

by defining the intrawall interaction as described for SWCNTs. For the interwall interaction, we can fix the hopping coefficients as

$$
\begin{aligned}
\tilde{\gamma}_{i j}(\boldsymbol{B})= & \beta \cos \vartheta_{i j} \exp \left(\frac{d_{i j}-a}{\delta}\right) \\
& \times \exp \left[\mathrm{i} \frac{2 \pi}{\Phi_{0}} \boldsymbol{d}_{i j} \cdot \boldsymbol{A}_{\boldsymbol{B}}\left(\frac{\boldsymbol{r}_{j}+\boldsymbol{r}_{i}}{2}\right)\right],
\end{aligned}
$$

where $\beta=\gamma_{0} / 8, a=3.34 \AA, \delta=0.45 \AA$, and $\vartheta_{i j}$ and $d_{i j}$ stand for the angle and the absolute distance between the two $\pi$ orbitals $\langle\langle i, j\rangle\rangle$ centered at positions $\boldsymbol{r}_{i}$ and $\boldsymbol{r}_{i}$ belonging to two different shells. ${ }^{60,61}$

As a representative example, the butterfly of a $(6,6) @(11,11)$ DWCNT is displayed in Fig. 11. For the parallel magnetic field, the interwall interaction gives rise to features at two levels: looking at individual bands, one can observe van Hove singularities crossing and sometimes avoid a crossing. The complex rules whether a crossing is avoided are not obvious from studying the butterfly plot only, but can be deduced by looking at the band structure and taking into account the various symmetries of the system. At larger scale in the butterfly plot, one finds a modulation of the pattern crossing from $E=E_{\mathrm{F}}-8 \mathrm{eV}$ at $B_{\|}=0$ to $E=E_{\mathrm{F}}$ $+8 \mathrm{eV}$ at $B_{\|} \approx 45 B_{0}^{\|}$.

To understand this phenomenon, we have studied a single unit cell in a magnetic field perpendicular to the plane of the resulting concentric ring (Fig. 12). The spectrum shows a periodic behavior of the modulation with a period $B_{0}^{\text {interwall }}$ $=\Phi_{0} /\left(d_{\mathrm{CC}} \delta r_{\text {interwall }} / 4\right)$ relating to the area of the minimal circular path between both walls. This period and the shape of the modulation are independent of the diameter of the DWCNT. A physical explanation for this modulation is as follows. Like the interaction in a two-atomic molecule, splitting two atomic orbitals into a bonding and an antibonding molecular orbital, the interwall interaction may also hybridize SWCNT states of the same energy into bonding and antibonding DWCNT states. However, the system has an approximate rotational symmetry, so the interwall interaction may only hybridize states of the same angular momentum.

At zero magnetic field, the angular momentum of the states at the bottom of the spectrum is zero in both shells. This allows hybridization, causing a split in the hybrid spectrum. At the upper end of the spectrum, the angular momentum does not match, prohibiting a hybridization. This is the cause for the strong electron-hole asymmetry visible in DWCNT butterfly plots.

By switching on a parallel magnetic field, the effective angular momentum is shifted by the Aharonov-Bohm phase gathered on a circular path around the tube. This shift depends on the cross section of the path, so it is different for the two shells. Therefore, the energy at which both angular momenta match depends on the magnetic field, causing the splitting region to travel over the energy range, which leads to the visible modulation in the parallel field butterfly plots.

For a simplified model - a double-wall square lattice tube-the modulation does follow a single cosine-shaped curve, as shown in the upper panel of Fig. 12. In comparison, the DWCNT shows an additional complexity: the underlying honeycomb lattice of graphene has a unit cell containing two atoms, resulting in two intertwined cosine curves, the second just becoming visible at the edge of Fig. 11.

For fields perpendicular to the axis of a DWCNT, the only large-scale effect caused by the interwall interaction observable in the butterfly plot is the hybridization-induced splitting already described for zero field. With growing field, this effect disappears, and the plot shows no remarkable global patterns.

\section{CONCLUSIONS}

The magnetic spectrum of two-dimensional infinite lattice electrons gives rise to the well-known Hofstadter butterfly. In this paper, we have shown that quasi-one-dimensional lattice electrons exhibit a spectrum which does resemble the fractal structure of the Hofstadter butterfly but with a finite cutoff due to the transversal confinement. We have calculated such pseudofractals for carbon nanotubes, a material at the focus of many nanoelectronic studies also in relation to the presence of external magnetic fields. We have calculated the density of states (butterfly plots) of several single wall carbon nanotubes and we could show (i) the strong dependence of the magnetic spectrum on the underlying chiral indices; (ii) the emergence of the graphene Hofstadter butterfly at increasing nanotube diameter. In particular, perpendicular fields induce an aperiodic and pseudofractal magnetic spec- 
trum. Periodic structures have been obtained for graphene ribbons, demonstrating that the aperiodicity of the perpendicular field butterfly plots is due to the incommensurability of the magnetic flux captured by elementary (hexagon) plaquettes of a CNT oriented at different angles towards the external field.

By studying the angle-resolved electronic structure of a SWCNT one can observe the emergence of snake states already predicted for nonuniform magnetic fields in a Hall bar. ${ }^{48}$ In our case, we have been able to devise an analytical model for the states at the top and the bottom of the energy spectrum by means of an effective mass approximation. In this latter case a continuum theory can capture the striping of the wave function along the region of the tube with zero normal field. Inversely, near the Fermi level, one cannot bypass the Dirac neutrino nature of the electronic states. We have interpreted the wave function striping by writing a Harper equation ${ }^{62}$ for square lattice electrons with a cylindrical geometry.

While the effects of parallel fields are of comparably simple nature in SWCNTs (being an expression of the Aharonov-Bohm oscillations due to a rigid shift of the graphene band structure sampled via the zone-folding method), this is not the case for DWCNTs. The electron-hole symmetry of $\pi$ bands in SWCNTs is broken once two shells are put in interaction. The resulting hybridization of inner and outer states could be clearly understood by means of two interacting Aharonov-Bohm rings.

Experimentally relevant effects have been calculated for SWCNTs of diameter of typical external shells in MWCNTs. There, underlying multifractal structure like that of Hofstadter can be observed already at a few tens of tesla, and an outstanding scaling law for the DOS at low magnetic fields near the Fermi energy has been given. The latter applies also to graphene ribbons and is intrinsically related to the massless dispersion at the charge neutrality point.

This study, though systematic, could not include very interesting issues which also deserve careful investigation, such as the effects of disorder on the butterfly plots of SWCNTs. Disordered SWCNTs can be thought in fact a model for the external shell of MWCNTs. More atomistically one could study the influence of the interwall interaction of the structure of large diameter DWCNTs (also reasonable models for MWCNTs).${ }^{63}$

\section{ACKNOWLEDGMENTS}

We acknowledge fruitful discussions with J. Kono, S. Krompiewski, E. Heller, U. Rößler, and C. Strunk. Ulrich Rößler made us aware of the interesting history of science involving Douglas Hofstadter, Gregory Wannier, and Gustav Obermeir which took place in Regensburg during the mid1970s and eventually led to the discovery of the Hofstadter butterfly thirty years ago. This work was funded by the Volkswagen Foundation under Grant No. I/78 340, by the European Union grant CARDEQ under Contract No. IST021285-2 and by the Deutsche Forschungsgemeinschaft within the Collaborative Research Center SFB 689. Support from the Vielberth Foundation is also gratefully acknowledged.

\section{APPENDIX A: HISTOGRAM METHOD}

A histogram method is the simplest method to get the magnetic spectrum of a quasi-1D system. It is also very efficient if the complete energy range has to be calculated. Starting from a periodic Hamiltonian of the form:

$$
\mathcal{H}=\left(\begin{array}{cccccc}
\ddots & \ddots & \ddots & & & \\
& H_{1}^{\dagger} & H_{0} & H_{1} & & \\
& & H_{1}^{\dagger} & H_{0} & H_{1} & \\
& & & \ddots & \ddots & \ddots
\end{array}\right),
$$

one can use Bloch theorem to get an effective Hamiltonian:

$$
H_{\text {eff }}(k)=H_{0}+e^{\mathrm{i} k a} H_{1}+e^{-\mathrm{i} k a} H_{1}^{\dagger}
$$

where $a$ is the length of the unit cell. Numerically scanning the 1D Brillouin zone $-\frac{\pi}{a}<k \leqslant \frac{\pi}{a}$ with a uniform distribution, one can now diagonalize the finite matrix $H_{\text {eff }}(k)$ for each value $k$. The resulting eigenvalues from this diagonalization are counted in a linear histogram over the full energy range and normalized to the total number of states. Depending on the resolution of the $k$ sampling, this histogram will become an arbitrarily good approximation to the density of states. Figures 1, 6, and 7 (CNT panels), 4 and 11 were calculated using this method.

The calculation of the data in Fig. 10 was heavily optimized by using an adaptive $k$ sampling in combination with a linear interpolation to reduce the number of diagonalizations in regions of smooth band structure and increase the precision at band edges.

\section{APPENDIX B: GREEN FUNCTION METHOD}

Another, more flexible method is that using Green functions: The bulk Green function $\mathcal{G}(E)$ of the infinite CNT can be calculated very efficiently by the following method. ${ }^{64}$

The periodic Hamiltonian in Eq. (A1) is used as the starting point of a recursive decimation scheme:

$$
\begin{aligned}
& H_{0}^{(0)}(E)=H_{0}, \\
& H_{01}^{(0)}(E)=H_{1}, \\
& H_{10}^{(0)}(E)=H_{1}^{\dagger} .
\end{aligned}
$$

With each recursion, the length of the effective unit cell is now doubled by decimating out every second cell:

$$
\begin{aligned}
H_{0}^{(n+1)}(E)= & H_{0}^{(n)}(E)+H_{01}^{(n)} \gamma^{(n)} H_{10}^{(n)}+H_{10}^{(n)} \gamma^{(n)} H_{01}^{(n)}, \\
& H_{01}^{(n+1)}(E)=H_{01}^{(n)} \gamma^{(n)} H_{01}^{(n)}, \\
& H_{10}^{(n+1)}(E)=H_{10}^{(n)} \gamma^{(n)} H_{10}^{(n)},
\end{aligned}
$$

where $\gamma^{(n)}=\left(E+\mathrm{i} \eta-H_{0}^{(n)}\right)^{-1}$ and $\eta$ is a small positive numerical value, chosen smaller than the desired energy resolution but large enough to provide fast convergence and numerical stability. 
Convergence is reached for $n \geqslant n^{\prime}$ if $\left\|H_{01}^{\left(n^{\prime}\right)}(E)\right\|+\| H_{01}^{\left(n^{\prime}\right)}$ $\times(E) \|<\epsilon$ for some matrix norm $\|\cdot\|$ and some small cutoff $\epsilon$. We can then retrieve the bulk Green function from the converged $H_{0}^{n^{\prime}}$ as

$$
\mathcal{G}_{\text {bulk }}(E) \approx\left(E+\mathrm{i} \eta-H_{0}^{\left(n^{\prime}\right)}\right)^{-1} .
$$

With the original Hamiltonian (A1) expressed in a $\pi$-orbital tight-binding basis, the resulting Green function $\mathcal{G}_{\text {bulk }}$ is a matrix in the same atomic basis of one unit cell. Therefore, the local density of states in each atom is directly given by

$$
\rho_{\mathrm{LDOS}_{i}}(E)=-\frac{1}{\pi} \operatorname{Im}\left[\mathcal{G}(E)_{i i}\right]
$$

summing up to the $\rho_{\mathrm{DOS}}(E)=\sum_{i} \rho_{\mathrm{LDOS}_{i}}(E)$. In the same run, the surface Green functions $\mathcal{G}_{\mathrm{S}}^{\mathrm{L} / \mathrm{R}}(E)$ can be used to calculate the transmission through the system using the Fisher-Lee relation $^{38}$ with a single unit cell selected as conductor, as shown in Fig. 3.

The Green function method and the histogram method both give numerical approximations to the same mathematical quantity, but their numerical errors are very different: while the former method tends to give fluctuations that show up as grainy structure in flat areas of the butterfly plot, the latter suffers from sampling problems around van Hove singularities. Both errors have to be countered with very high resolution scanning and down sampling of the data. The data presented in the figures of this article typically took several hours to weeks of computation time on standard PCs [Intel(R) Pentium(R) 4, $3 \mathrm{GHz}$.

The work presented here was done using the following Open Source ${ }^{65}$ (R) software: Python as programming language, ${ }^{66}$ NumPy (Refs. 67 and 68) and SciPy (Ref. 69) for numerical computations, PyTables for data storage and handling, ${ }^{70}$ matplotlib for data visualization, ${ }^{71}$ inkscape for figure preparation, ${ }^{72}$ and TeXmacs for authoring. ${ }^{73}$
${ }^{1}$ D. R. Hofstadter, Phys. Rev. B 14, 2239 (1976).

${ }^{2}$ S. Iijima, Nature (London) 354, 56 (1991).

${ }^{3}$ A. Oberlin, M. Endo, and T. Koyama, J. Cryst. Growth 32, 335 (1976).

${ }^{4}$ M. S. Dresselhaus, G. Dresselhaus, and P. C. Eklund, Science of Fullerenes and Carbon Nanotubes (Academic Press, San Diego, 1996).

${ }^{5}$ R. Saito, Physical Properties of Carbon Nanotubes (World Scientific Publishing Company, London, 1998).

${ }^{6}$ S. Reich, C. Thomsen, and J. Maultzsch, Carbon Nanotubes: Basic Concepts and Physical Properties (Wiley-VCH, Weinheim, 2004).

${ }^{7}$ E. Thune and C. Strunk, Quantum Transport in Carbon Nanotubes, Lecture Notes in Physics Vol. 680 (Springer, Berlin, 2005), pp. 351-380.

${ }^{8}$ H. W. C. Postma, T. Teepen, Z. Yao, M. Grifoni, and C. Dekker, Science 293, 76 (2001)

${ }^{9}$ S. Frank, P. Poncharal, Z. L. Wang, and W. A. de Heer, Science 280, 1744 (1998).

${ }^{10}$ F. Wang, G. Dukovic, L. E. Brus, and T. F. Heinz, Science 308, 838 (2005)

${ }^{11}$ H. Ajiki and T. Ando, J. Phys. Soc. Jpn. 62, 1255 (1993).

${ }^{12}$ S. Zaric, G. N. Ostojic, J. Kono, J. Shaver, V. C. Moore, M. S. Strano, R. H. Hauge, R. E. Smalley, and X. Wei, Science 304, 1129 (2004).

${ }^{13}$ A. Bachtold, C. Strunk, J.-P. Salvetat, J.-M. Bonard, L. Forró, T. Nussbaumer, and C. Schönenberger, Nature (London) 397, 673 (1998).

${ }^{14}$ E. D. Minot, Y. Yaish, V. Sazonova, and P. L. McEuen, Nature (London) 428, 536 (2004).

${ }^{15}$ H. Ajiki and T. Ando, J. Phys. Soc. Jpn. 62, 2470 (1993).

${ }^{16}$ R. Saito, G. Dresselhaus, and M. S. Dresselhaus, Phys. Rev. B 50, 14698 (1994).

${ }^{17}$ H. Ajiki and T. Ando, J. Phys. Soc. Jpn. 65, 505 (1995).

${ }^{18}$ S. Roche and R. Saito, Phys. Rev. Lett. 87, 246803 (2001).

${ }^{19}$ G. Fedorov, B. Lassagne, M. Sagnes, B. Raquet, J.-M. Broto, F.
Triozon, S. Roche, and E. Flahaut, Phys. Rev. Lett. 94, 066801 (2005).

${ }^{20}$ Y.-W. Son, J. Ihm, M. L. Cohen, S. G. Louie, and H. J. Choi, Phys. Rev. Lett. 95, 216602 (2005).

${ }^{21}$ S. Roche, G. Dresselhaus, M. S. Dresselhaus, and R. Saito, Phys. Rev. B 62, 16092 (2000).

${ }^{22}$ B. Stojetz, C. Miko, L. Forró, and C. Strunk, Phys. Rev. Lett. 94, 186802 (2005).

${ }^{23}$ B. Stojetz, S. Roche, C. Miko, F. Triozon, L. Forró, and C. Strunk, cond-mat/0607034 (unpublished).

${ }^{24}$ R. Rammal, J. Phys. (Paris) 46, 1345 (1985).

${ }^{25}$ C. Kreft and R. Seiler, J. Math. Phys. 37, 5207 (1996).

${ }^{26}$ T. G. Pedersen, Phys. Rev. B 68, 245104 (2003).

${ }^{27}$ D. Osadchy and J. E. Avron, J. Math. Phys. 42, 5665 (2001).

${ }^{28}$ Y. Iye, E. Kuramochi, M. Hara, A. Endo, and S. Katsumoto, Phys. Rev. B 70, 144524 (2004).

${ }^{29}$ J. Brüning, V. V. Demidov, and V. A. Geyler, Phys. Rev. B 69, 033202 (2004).

${ }^{30}$ J. G. Analytis, S. J. Blundell, and A. Ardavan, Am. J. Phys. 72, 613 (2004)

${ }^{31}$ C. Zhou, M. Berciu, and R. N. Bhatt, Phys. Rev. B 71, 125310 (2005).

${ }^{32}$ C. Albrecht, J. H. Smet, K. von Klitzing, D. Weiss, V. Umansky, and H. Schweizer, Phys. Rev. Lett. 86, 147 (2001).

${ }^{33}$ F. Herlach, Rep. Prog. Phys. 62, 859 (1999).

${ }^{34}$ R. Peierls, Z. Phys. 80, 763 (1933).

${ }^{35}$ L. V. Hove, Phys. Rev. 89, 1189 (1953).

${ }^{36}$ S. Datta, Quantum Transport: Atom to Transistor (Cambridge University Press, Cambridge, U.K., 2005).

${ }^{37}$ G. Cuniberti, F. Großmann, and R. Gutiérrez, The Role of Contacts in Molecular Electronics (Springer, Berlin, 2002), Vol. 42, pp. 133-149.

${ }^{38}$ D. S. Fisher and P. A. Lee, Phys. Rev. B 23, 6851 (1981).

${ }^{39}$ S. Krompiewski, J. Martinek, and J. Barnaś, Phys. Rev. B 66, 073412 (2002).

${ }^{40}$ S. Krompiewski, R. Gutiérrez, and G. Cuniberti, Phys. Rev. B 69, 
155423 (2004)

${ }^{41}$ V. Moldoveanu, A. Aldea, A. Manolescu, and M. Niță, Phys. Rev. B 63, 045301 (2001).

${ }^{42}$ K. S. Novoselov, A. K. Geim, S. V. Morozov, D. Jiang, M. I. Katsnelson, I. V. Grigorieva, S. V. Dubonos, and A. A. Firsov, Nature (London) 438, 197 (2005).

${ }^{43}$ Y. Zhang, Y.-W. Tan, H. L. Stormer, and P. Kim, Nature (London) 438, 201 (2005).

${ }^{44}$ C. Berger, Z. Song, X. Li, X. Wu, N. Brown, C. Naud, D. Mayou, T. Li, J. Hass, A. N. Marchenkov, E. H. Conrad, P. N. First, and W. A. de Heer, Science 312, 1191 (2006).

${ }^{45}$ H.-W. Lee and D. S. Novikov, Phys. Rev. B 68, 155402 (2003).

${ }^{46}$ Y. Zheng and T. Ando, Phys. Rev. B 65, 245420 (2002).

${ }^{47}$ F. D. M. Haldane, Phys. Rev. Lett. 61, 2015 (1988).

${ }^{48}$ J. E. Müller, Phys. Rev. Lett. 68, 385 (1992).

${ }^{49}$ V. A. Margulis and M. A. Pyataev, Phys. Rev. B 72, 075312 (2005).

${ }^{50}$ D. V. Zav'yalov, S. V. Kryuchkov, and N. E. Meshcheryakova, Phys. Solid State 47, 1169 (2005).

${ }^{51}$ K. Handrich, Phys. Rev. B 72, 161308(R) (2005).

${ }^{52}$ A. Krakovsky, Phys. Rev. B 53, 8469 (1996).

${ }^{53}$ J. Yi, M. Porto, and G. Cuniberti, in Encyclopedia of Nanoscience and Nanotechnology, edited by H. S. Nalwa (American Scientific Publishers, Stevenson Ranch, CA, 2004), Vol. 5, pp. 607617.

${ }^{54}$ E. Perfetto, J. González, F. Guinea, S. Bellucci, and P. Onorato, cond-mat/0604046 (unpublished).

${ }^{55}$ S. Zaric, G. N. Ostojic, J. Shaver, J. Kono, O. Portugall, P. H. Frings, G. L. J. A. Rikken, M. Furis, S. A. Crooker, X. Wei, V. C. Moore, R. H. Hauge, and R. E. Smalley, Phys. Rev. Lett. 96, 016406 (2006).

${ }^{56} \mathrm{~J}$. Kono (private communication).

${ }^{57}$ J. L. Hutchisona, N. A. Kiselev, E. P. Krinichnayac, A. V. Kres- tininc, R. O. Loutfyd, A. P. Morawskyc, V. E. Muradyanc, E. D. Obraztsovae, J. Sloanf, S. V. Terekhove, and D. N. Zakharovc, Carbon 39, 761 (2001).

${ }^{58}$ T. Sugai, H. Yoshida, T. Shimada, T. Okazaki, and H. Shinohara, Nano Lett. 3, 769 (2003).

${ }^{59}$ Y. H. Ho, G. W. Ho, S. J. Wu, and M. F. Lin, J. Vac. Sci. Technol. B 24, 1098 (2006).

${ }^{60}$ P. Lambin, J. Charlier, and J. Michenaud, Electronic Structure of Coaxial Carbon Tubules (World Scientific, Singapore, 1994), pp. 130-134.

${ }^{61}$ S. Roche, F. Triozon, A. Rubio, and D. Mayou, Phys. Rev. B 64, 121401(R) (2001)

${ }^{62}$ P. G. Harper, Proc. Phys. Soc., London, Sect. A 68, 874 (1955).

${ }^{63}$ N. Nemec and G. Cuniberti (unpublished).

${ }^{64}$ M. P. López Sancho, J. M. López Sancho, and J. Rubio, J. Phys. F: Met. Phys. 15, 851 (1985).

${ }^{65}$ N. Bezroukov, First Monday 4, 10 (1999).

${ }^{66}$ Python Reference Manual, edited by G. van Rossum and F. Drake (PythonLabs, VA, 2001), http://www.python. org.

${ }^{67}$ D. Ascher, P. Dubois, K. Hinsen, J. Hugunin, and T. Oliphant, computer code NUMERICAL PYTHON 2001, http:// www.numpy.org.

${ }^{68}$ T. E. Oliphant, Guide to NumPy (Trelgol Publishing, Spanish Fork, UT, 2006), http://www.numpy.org.

${ }^{69}$ E. Jones et al., computer code SCIPY, 2001, http://www.scipy. org.

${ }^{70}$ F. Altet, I. Vilata, S. Prater, V. Mas, T. Hedley, A. Valentino, and J. Whitaker, computer code PYTABLES, 2002, http:// www.pytables.org.

${ }^{71}$ P. Barrett, J. Hunter, and P. Greenfield, in Astronomical Data Analysis Software \& Systems XIV. (2004), http://matplotlib. sourceforge. net

${ }^{72}$ Computer code INKSCAPE, 2003, http://www.inkscape.org

${ }^{73}$ J. van der Hoeven, Cah. Gutenberg 39-40, 39 (2001). 